\title{
El tutor de prácticas y su influencia en la for- mación de profesionales en los Grados de Educación
}

\section{The tutor of practices and his professionals influence in the Education Degrees}

\author{
Ascensión Palomares Ruiz \\ Universidad de Castilla La Mancha (UCLM). \\ Albacete (España) \\ ascension.palomares@uclm.es
}

María del Carmen Alarcón Palomares CEIP Santa Ana (Cuenca). Junta de Comunidades de Castilla-La Mancha (España)

mariacalarpa@hotmail.com

Fecha de recepción 27-04-2018

Fecha de aceptación 10-03-2018

Palomares Ruiz, A. y Alarcón Palomares, M.C. (2018). El tutor de prácticas y su influencia en la formación de profesionales en los Grados de Educación. Revista Prácticum, 3(1), 34-47. 


\section{Resumen}

Actualmente, los problemas educativos ocupan un papel fundamental de reflexión social, por lo que resulta preciso potenciar el conocimiento y conseguir una educación obligatoria de calidad que garantice los derechos humanos, la igualdad entre los géneros, la promoción de una cultura de paz y no violencia, la ciudadanía mundial, la valoración de la diversidad cultural y de la contribución de la cultura al desarrollo sostenible. Todo ello, exige un importante cambio en la formación inicial y continua, con valores y emprendimiento inteligente. Esta investigación se ha desarrollado en los Grados de Educación de la Facultad de Educación de Albacete del Centro Asociado de la UNED en los cursos 2015-16 y 2016-17. El principal objetivo se ha centrado en analizar la situación actual para optimizar la formación práctica de los/as Maestros/as, los/as Pedagogos/as y los/as Educadores/as Sociales, y poder enriquecerla con las aportaciones de mejora y las evaluaciones que realizan todos los intervinientes en el proceso formativo (alumnado, profesionales colaboradores, profesorado, instituciones, etc.). Los resultados muestran que los/as tutores/as (profesionales colaboradores) valoran la utilidad de las prácticas profesionales en la formación de los Grados, estimando que son fundamentales, para establecer un acercamiento y proporcionar un aprendizaje que no se produce en la Facultad a nivel teórico. Acerca de la duración del periodo de prácticas se valora que la duración estipulada debería ampliarse. Como conclusión más significativa resulta preciso subrayar la falta de consideración y reconocimiento académico de las prácticas profesionales, así como la insuficiente relación entre la teoría y la práctica, en la formación de los profesionales estudiados, como aspectos destacados por todos los participantes en la investigación.

\section{Abstract}

Nowadays, educational problems are a fundamental issue of social reflection, so it is essential to develop the knowledge and achieve an obligatory education of quality which guarantees the human rights, gender equality, promotion of a culture of peace and no violence, a global citizenship, and the appreciation of the cultural diversity and the contribution of the culture to the sustainable development. All these things require an important change in the pre-service and in-service training, with values and an intelligent task. This research has been developed in the Education Degrees of the Education Faculty, UNED Associate Center of Albacete, 2015-16 and 2016-17 courses. The main objective has been focused to the support and strengthening of teaching, to optimize the formation of teachers, Pedagogues, and Social Educators, and to enrich them with the contributions and improvement evaluations made by all people involved in the training process (students, collaborators, faculties, institutions, etc.). The results show that tutors (collaborating professionals) value the usefulness of the professional practices in the Degrees, considering that they are essential, to get an approach and to provide a learning that is not only produced in the Faculty on a theoretical level but also in a practical level. Related to the duration of the period of practices, it is valued that it should be extended. The most meaningful conclusion, we can highlight the lack of consideration and academic acknowledgement of the Practicum, as well as the insufficient link between theory and practice, in the formation of the studied professionals and the underlined aspects by all the participants in the research. 


\section{Palabras clave}

Tutor/a, Competencias profesionales, Calidad educativa, Sociedad inclusiva, Practicum.

\section{Keywords}

Tutor, Professional competences, Educational quality, Inclusive society, Practicum.

\section{Introducción}

Vivimos en un mundo caracterizado por conflictos y crisis, nacionalismo y fanatismo, problemas y desafíos medioambientales; por ello, la Educación para la Ciudadanía Mundial (ECM) resulta un importe desafío. Motivadamente, tiene dimensiones individuales y sociales y se propone empoderar a los educandos para que asuman papeles activos a fin de hacer frente y resolver retos mundiales, así como para devenir agentes proactivos que contribuyan a crear un mundo más pacífico, tolerante, inclusivo y seguro. La Agenda y el Marco de Acción 2030 para la Educación, especialmente la meta 4.7, apela a los países para que garanticen los derechos humanos, la igualdad entre los géneros, la promoción de una cultura de paz y no violencia, la ciudadanía mundial y la valoración de la diversidad cultural y de la contribución de la cultura al desarrollo sostenible, entre otros medios (UNESCO, 2017). Todo ello, exige una formación de los profesionales de la educación, con competencias y actitudes para dar respuestas creativas a los retos del complejo y cambiante mundo actual. Por tanto, como indica Juliá (2017), resulta inaplazable dotar a los sistemas educativos universitarios de planes que garanticen su suficiencia financiera para preservar la igualdad de oportunidades, una mayor autonomía y capacidad de gestión, con una mayor conexión entre su sistema educativo con el tejido productivo. Todo ello, exige un importante cambio en la formación práctica de los profesionales de la educación con valores y emprendimiento inteligente.

Resulta importante subrayar que la educación europea está sufriendo grandes transformaciones que intentan superar los desajustes internos y externos. Además, en todos los sistemas educativos, aunque con diferente forma e intensidad, se destaca: 
a) La necesidad de una formación práctica que empodere al alumnado para afrontar los retos de la sociedad actual.

b) Los malos rendimientos de la educación secundaria obligatoria.

c) La insuficiente adaptación entre el sistema educativo y el productivo.

d) La incorporación de los avances tecnológicos, con la aparición de nue vas formas de cultura y sociabilidad.

e) Los crecientes comportamientos violentos, tanto en los centros edu cativos como en la sociedad en general. (Domínguez, Álvarez \& López, 2017)

Razonadamente, los problemas educativos ocupan, en los países europeos, un papel fundamental de reflexión social, por lo que se pretende potenciar el conocimiento y conseguir una educación obligatoria de calidad, que posibilite el desarrollo económico e incremente el bienestar social. Como especifica Cortina (2017), el sistema educativo debe propiciar el diálogo intercultural que forme a los ciudadanos para respetar las culturas, porque no se puede renunciar a la riqueza que una cultura puede aportar; pero, a la vez, ese respeto tiene que llevar a un diálogo para que los ciudadanos puedan discernir qué valores y costumbres merecen la pena reforzar y cuáles obviar.

Los problemas provocados por las desigualdades y desequilibrios sociales se extienden por todos los países, resaltando especialmente en los sistemas educativos, por lo que se precisa un debate abierto -y con amplia participación social- sobre el futuro de la educación, la Escuela y los sistemas educativos, tanto en España como en la UE. Consecuentemente, es imprescindible -y urgente- una nueva consideración de la educación en la UE, que pueda coordinar todos los espacios educativos, que preste más atención al profesorado, que posibilite la formación durante toda la vida, que se adapte con eficacia a los necesarios cambios de métodos, objetivos, contenidos, etc., y que favorezca la igualdad de oportunidades y la integración de todos y todas, sin discriminaciones de ningún tipo. Estos problemas, no son nuevos; pero lo novedoso es que se han convertido en comunes para todos los ciudadanos europeos. En este sentido, la Declaración de la UNESCO sobre la educación superior en el siglo XXI (1998), señala que las instituciones de educación superior deben formar a los estudiantes para que se conviertan en ciudadanos bien informados y profundamente motivados, provistos de un sentido crítico y capaces de analizar los problemas de la sociedad, buscar soluciones, aplicarlas y asumir responsabilidades sociales.

Igualmente, el proceso de convergencia europeo supone un importante cambio en el planteamiento de la docencia en las Universidades españolas, por lo que se tendría que modificar el actual sistema. La redefinición de las metodologías supone que el alumnado tendrá que adquirir un aprendizaje que abarque el conocimiento científico de su carrera y, además, diversas capacidades y destrezas. Actualmente, existe un cierto consenso sobre los contenidos de formación, en el sentido en que se han de incluir diferentes ámbitos de conocimiento, así como en sus contribuciones a la comprensión de la enseñanza y el desarrollo de diversas capacidades requeridas por las tareas y responsabilidades profesionales. Sin embargo, no existe el mismo consenso a la hora de clarificar qué representa y en qué consiste una enseñanza de calidad y una enseñanza efectiva para que los alumnos aprendan significativamente. 
Resulta evidente que han de aprender diversos tipos de contenidos y desarrollar con ellos diferentes competencias. Por ello, además de dominar dichos contenidos y las metodologías relativas a las materias del currículo con la idea de que los alumnos adquieran aprendizajes relevantes, el docente también tiene que tener en cuenta criterios morales. El éxito de la enseñanza depende de lo que el profesor sabe, de cómo lo enseña y de sus intenciones moralmente defendibles. Ello exige conocer sus formas de pensar y aprender, sus intereses, motivaciones, etc., dado que la profesión docente no llega a ser exitosa sin la complicidad de aquéllos para quienes se ejerce. El profesorado ha de conectar con el alumnado, no sólo con la cabeza, sino también con registros personales y relaciones positivas (Pérez Gómez, 2003). Por ello, se tendrá que conocer la dinámica de trabajo en clase, los factores influyentes para la creación de un buen entorno motivador, el conocimiento y uso inteligente de estrategias pedagógicas y materiales variados, etc.

No se debe olvidar que la enseñanza y el aprendizaje son fenómenos singulares y altamente imprevisibles; por ello, a fin de que el docente no se sienta desbordado, tiene que tener muy claro su plan de trabajo $y$, al tiempo, poseer una actitud reflexiva y flexible para ajustarlo según el desarrollo de los acontecimientos. Ello le exige gozar de conocimientos y procedimientos adecuados para conocer la realidad personal y el entorno al que pertenecen sus estudiantes, seguir el curso de sus aprendizajes e ir recabando la información precisa para evaluar no sólo al alumnado sino también su propio trabajo, convirtiéndolo en objeto de revisión, análisis reflexivo e -incluso- una fuente de aprendizaje, comprendiendo lo que sucede y obteniendo lecciones para el futuro. Consecuentemente, con fines de mejora, en la formación de los profesionales de la educación hay que trabajar también contenidos y procesos de trabajo relacionados con la investigación sobre la propia práctica (Palomares \& Serrano, 2016).

Lógicamente, como indican De Lara y Quintanal (2006), la calidad de un programa de formación no reside en la cantidad de contenidos, sino en su relevancia para el currículo escolar y la enseñanza, en el grado de articulación y coherencia que logre entre los diferentes componentes (contenidos, metodologías, teoría y práctica). Los docentes deben adquirir aprendizajes de carácter cognitivo (comprender los factores implicados en la enseñanza y aprendizaje y desarrollar habilidades relacionadas con la planificación, enseñanza, evaluación), de carácter personal (conferir sentido y propósitos a la profesión, analizar, reflexionar y mejorar las propias ideas y prácticas), de carácter social, cómo aprender a trabajar con sus compañeros, participar en la vida y tareas del centro, así como establecer y sostener relaciones adecuadas con otros agentes que incidan en la educación del alumnado (familias u otros profesionales). Además, la profesión requiere una dimensión moral, el desarrollo de actitudes y disposiciones para actuar adecuadamente de acuerdo con unos valores y principios que le faciliten aprendizajes de carácter ético.

Si nos centramos en nuestro país, el núcleo de la propuesta formativa de los Grados de Pedagogía, Grados de Maestro y Educación Social, se especifican unas competencias genéricas que se reiteran en las competencias enunciadas en cada uno de los módulos que -en la práctica- esconden las disciplinas (psicología, pedagogía, sociología, materias de la especialidad y didáctica de estas, innovación, Prácticum y Memoria final de Grado) (ANECA, 2005). 
Razonadamente, estimamos que, los valores básicos que deben impregnar todo el proceso formativo de las titulaciones estudiadas en esta investigación y en las competencias a desarrollar, son:

a) Compromiso y dedicación a la profesión.

b) Consideración y respeto de la diversidad.

c) Creencia en que todas las personas pueden aprender.

d) Colaboración y disposición por compartir.

e) Espíritu de equipo.

f) Implicación (pasión) en el aprendizaje continuado y la búsqueda de la excelencia profesional.

En resumen, sería imprescindible que todos los profesionales ostentaran: flexibilidad, constancia, cordura, juicio, saber estar, compromiso, justicia, etc. Por ello, estimamos que se debe favorecer más la formación de profesionales reflexivos y críticos que den respuesta a las necesidades de los usuarios, sin ningún tipo de discriminación y comprometidos con la justicia social.

Consecuentemente, las propuestas que se engendren de la investigación realizada sobre la formación de los profesionales estudiados no será una solución mágica; pero sí merece considerarse como una de las llaves importantes para crear ideas y propósitos con sentido, desarrollar conocimientos y capacidades. En la sociedad actual, cada vez más injusta e insolidaria, sin una formación buena y efectiva, la brecha entre las intenciones y las prácticas, entre la deseable implicación de los estudiantes en su propia formación y el desenganche, entre los objetivos imprescindibles y los resultados, muy probablemente, tenderá a aumentar, si no se adoptan las medidas adecuadas en tiempo y forma.

Resulta evidente que se deben revisar a fondo los métodos de la formación, unir la teoría y la práctica, establecer vínculos entre los centros universitarios y los centros educativos e instituciones colaboradoras, imprimir a las experiencias de formación un carácter mucho más vivencial y clínico, centrado en la reconstrucción de supuestos o creencias indebidas, de forma que sean una oportunidad para hablar, observar, analizar situaciones, problemas y tareas concretas, aprender analizando las buenas prácticas e investigando críticamente sobre procesos y resultados.

En la sociedad actual, se debe defender la utopía social y educativa, considerando la educación como una herramienta para el cambio y la transformación social hacia modelos más democráticos y justos (Palomares, 2013). Por ello, la formación de los profesionales de la educación debe ser una reflexión en y sobre la acción, que les facilite claves de justicia y equidad, como un medio para conseguir una sociedad con una ciudadanía más feliz y educada, luchando preventivamente contra la exclusión educativa y social. 
Lógicamente, para conseguir una educación democrática y equitativa, también se deben desarrollar conocimientos y capacidades de análisis y crítica desde el conocimiento de la realidad, que permitan conocer dónde se encuentran las barreras contra el derecho a la educación, por qué y de qué manera las políticas educativas pueden obstruir el diseño y desarrollo de un proyecto educativo.

La formación de los profesionales de la educación debe estar centrada en la adquisición y desarrollo de los conocimientos y capacidades que son necesarios para un buen ejercicio de su profesión, logrando que adquieran las oportunidades precisas para conseguir las herramientas conceptuales y prácticas adecuadas. Una vez más, conviene resaltar la necesidad de reconocer y valorar la singularidad de cada estudiante $y$, por tanto, su diversidad $y$, consecuentemente, las diversidades que se presentan en el Centro y en el aula. Por ello, resulta fundamental el tipo y calidad de los vínculos personales y sociales que se establecen en el proceso formativo, potenciando el respeto mutuo, cultivar la confianza, la exigencia del esfuerzo y responsabilidad, apoyar y buscar complicidades, que sirva de modelo personal y social de educación y civismo, sentirse responsable de todos y cada de las personas con las que se trabaja y resistir las tentaciones de echar balones fuera y asumir las responsabilidades que le correspondan a cada uno. Resulta evidente que, la ética del aprendizaje y desarrollo de la profesión no será posible si no existe un marco de valores y normas de la profesión que provoquen la disposición y el compromiso con la mejora de las propias ideas y práctica, como algo inherente a todo docente a lo largo de su vida. Sólo un profesional bien preparado y comprometido con la profesión y propósitos morales, está en condiciones de garantizar el derecho a una educación de calidad, sin ningún tipo de discriminación (Palomares, 2007).

Motivadamente, una figura fundamental en el proceso de enseñanza-aprendizaje de las prácticas profesionales a nivel universitario es la del tutor/a (Profesional colaborador). Como indican González, Álvarez y Fernández (2012), la tutoría ha de servir como eje principal desde el que atender la educación integral de la persona a través del desarrollo de valores como la solidaridad, empatía o el respeto, de cara a transmitir al alumnado los postulados de una sociedad globalizada en la que la diversidad cultural es una realidad clave. Por ello, los/as tutores/as se convierten en las personas encargadas de orientar y guiar al alumnado. Efectivamente, como figura orientadora debe poseer un amplio conocimiento actualizado en diversas esferas que describen integralmente al ser humano y que requieren de un esfuerzo continuo por conocer a las personas con las que trabajan $y$, al tiempo, comprender todo el proceso madurativo de referencia. En consecuencia, supone elaborar un esquema explicativo donde se integran variables de muy diversa índole en un complicado entramado de relaciones multidireccionales, con el objetivo de preservar una perspectiva que sea capaz de comprender todas las situaciones y particularidades posibles.

Razonadamente, se requiere una perspectiva fundada en el conocimiento y en los datos recogidos en un proceso de evaluación de forma tranquila y exitosa, donde se cimentarán también de forma integral todas las técnicas y procesos de intervención en orientación vocacional y donde, como hemos comentado, es imposible compartimentar un saber a expensas de las áreas contempladas en el trabajo del profesional, tales como factores personales, sociales, etc. 


\section{Metodología}

El principal objetivo de la investigación se ha centrado en el análisis de la realidad actual para la realización de propuestas que posibiliten mejorar la formación práctica del alumnado de los Grados estudiados. En este sentido, se ha pretendido perfeccionar la tarea docente en la línea indicada por la UNESCO (1999), analizando la relación entre la investigación científica, la educación, la innovación tecnológica y los beneficios prácticos, considerando que la realidad es mucho más diversificada y compleja. Por ello, ya no se puede justificar el progreso de la ciencia exclusivamente por la búsqueda de conocimientos y se precisa la justificación de su pertinencia y eficacia para responder a las necesidades y expectativas de la sociedad.

La investigación se ha desarrollado durante los cursos 2015-16 y 2016-07, con alumnado de los Grados de Maestro (especialidades de Educación Infantil y Educación Primaria) de la Facultad de Educación de Albacete (UCLM) y con alumnado de prácticas profesionales de Educación Social y de Pedagogía del Centro Asociado de la UNED en Albacete. Los objetivos específicos planteados son:

a) Identificar los elementos clave para el éxito en el diseño y desarrollo del Prácticum, potenciando la autonomía del alumnado, el pensamiento reflexivo y crítico, las actitudes colaborativas, las destrezas profesionales y la capacidad de auto y coevaluación.

b) Analizar las competencias que se ponen en juego para conseguir que el alumnado interactúe social y profesionalmente con su entorno.

c) Facilitar la reflexión del alumnado sobre lo que hace, cómo lo hace y qué resultados va obteniendo, para proponer -en su caso- acciones de mejora.

El modelo de investigación más adecuado para la consecución de los objetivos planteados es de carácter no experimental propiamente dicho (Campbell y Stanley, 1978), estando principalmente basado en la aplicación de cuestionarios.

La población objeto de estudio queda definida por el conjunto de estudiantes que cursan estudios de Grados de Maestro, Grado de Pedagogía y Grado de Educación Social en la provincia de Albacete. Igualmente, se ha considerado el conjunto de profesionales colaboradores en el Prácticum; sin embargo, la participación de éstos, al no constituir una muestra representativa de todas las instituciones, sólo ha permitido considerar las conclusiones sobre los aspectos más significativos de las prácticas profesionales y las propuestas de mejora.

El número total de alumnado participante en los dos años ha sido de 118 . Al mismo tiempo, el número de hombres y de mujeres que componen la muestra ha sido idéntico, circunstancia que ha incidido en el número total de la muestra, al estar matriculados en dichas titulaciones un número más elevado de mujeres. Asimismo, se ha obtenido respuesta de 24 profesionales colaboradores. 
La recogida de información se realizó a través de cuestionarios elaborados ad hoc (previamente validados) para esta investigación, que se fueron entregando a todos los participantes. Los cuestionarios pasados al alumnado y a personal profesional y colaborador, estaban integrados por 24 ítems valorados por una escala de tipo Likert de cuatro valores ( $1=$ nada; $2=$ Poco; $3=$ Suficiente; y $4=$ Mucho), han facilitado obtener -de forma sistemática y ordenada- información sobre las variables que intervienen en la investigación de la realidad en que nos encontramos y -al tiempo- provocar la reflexión sobre su propia actividad profesional, evaluar la importancia de las competencias profesionales y la percepción del logro de las mismas. Consecuentemente, se ha comprobado que la fiabilidad del instrumento es muy alta (Alpha de Cronbach .94).

De acuerdo con lo indicado por Guba (1989), se puede decir que la dimensión cualitativa de la investigación reúne los cuatro criterios de rigor relacionados con la credibilidad de la investigación: valor de verdad (credibilidad), aplicabilidad (transferibilidad), consistencia (dependencia) y neutralidad. Además, el rigor de la investigación está también garantizado, en lo que afecta a la dimensión cuantitativa de la investigación, la construcción de instrumentos, el control de todo el proceso y el rigor en la recogida de la información.

En la elaboración de los cuestionarios se ha considerado, entre otros, los siguientes aspectos:

a) Concreción de las variables del estudio, a partir de los objetivos planteados.

b) Establecimiento de criterios e indicadores.

c) Elaboración de cuestionarios abiertos y facilitadores de la reflexión crítica y la aportación del investigador y de los participantes en la misma.

d) Validación de los cuestionarios y su elaboración definitiva.

e) Aplicación de cuestionarios equivalentes para todos los participantes, introduciendo las mínimas modificaciones entre los dos grupos (profesionales colaboradores y alumnado).

Todos los cuestionarios se han dividido en grandes apartados, entre los que hay que destacar:

a) Datos generales, que incluyen las variables personales, el Centro donde se cursan los estudios y la institución en que se realiza el Prácticum.

b) Contexto socio-formativo, que se centra en las variables relativas a las competencias básicas de cada titulación universitaria y del contexto social en que realizan el Prácticum.

c) Las Prácticas Profesionales, en el diseño y desarrollo del Prácticum.

d) Propuestas de mejora para el proceso formativo, en general, y el Prácticum, en particular. 
Los cuestionarios utilizados se han pasado a las personas implicadas en el estudio (alumnado y profesionales colaboradores), con el fin de recopilar informaciones de carácter general, y han servido de base para la elaboración posterior de las entrevistas. Además, las entrevistas personales se han ejecutado a una muestra igual de profesionales colaboradores y de alumnado de cada Grado, siendo un total de 12 profesionales colaboradores y 12 estudiantes, la muestra ha sido elegida por interés de las investigadoras y seleccionado igual número de hombres que mujeres, con el fin de obtener información complementaria de determinados aspectos no recogidos en los cuestionarios.

Toda la información obtenida en los cuestionarios y las entrevistas se ha analizado en dos fases y con dos técnicas diferentes, siendo la primera de ellas de tipo cuantitativo y, la segunda, preferentemente cualitativa. El análisis cuantitativo ha tenido menor importancia, aunque ha posibilitado conocer:

a)Deficiencias formativas.

b)Los intereses del alumnado respecto a su proceso formativo.

c)Las diferencias y similitudes entre las tres titulaciones universitarias (Grados de Maestro, Grado de Pedagogía y Grado de Educación Social).

d)Las principales dificultades en el diseño y desarrollo del Prácticum.

e)La elaboración de propuestas de mejora, etc.

Los análisis descriptivos se han realizado mediante el programa SPSS 23.0, que han facilitado -en algunos casos- información muy concreta sobre los indicadores establecidos y el grado de cumplimiento de cada uno de ellos.

\section{Resultados}

El trabajo realizado ha permitido profundizar en las metodologías utilizadas en el proceso formativo de tres titulaciones universitarias y, más concretamente, en la consideración en el diseño y desarrollo del Prácticum.

La muestra seleccionada pretendía ser lo más representativa posible; sin embargo, al trabajar con alumnado de diferentes regímenes universitarios (presencial y a distancia), con una duración de dos cursos, con edades e intereses muy diferentes, etc., se ha provocado que se consideren más los resultados cualitativos y las propuestas, que los cuantitativos, ya que éstos -en todo caso- son simplemente orientativos. En cambio, la información recopilada posibilita un mejor trabajo y una mayor optimización en el diseño y desarrollo del Prácticum, en las titulaciones universitarias. 
En la valoración del diseño y desarrollo del Prácticum entre el alumnado de la UNED (Grado de Pedagogía y Grado de Educación Social), no se observan diferencias en los resultados, al ser prácticamente los mismos. Sin embargo, sí existen diferencias en la variable sexo, entre el alumnado del Grado de Maestro, dado que el $89 \%$ de las alumnas lo considera como fundamental en su formación, frente a un $73 \%$ de los chicos. El análisis detallado de estas variables nos conduce a la motivación intrínseca del alumnado, existiendo una alta relación entre la motivación de este y la valoración otorgada al Prácticum, dado que se ha otorgado la misma puntación en ambos indicadores. Los profesionales colaboradores han destacado, en un $100 \%$, la importancia del Prácticum y la reivindicación de un reconocimiento adecuado del mismo. Como propuestas más significativas y comunes a las tres titulaciones, considerando las respuestas de la escala de Likert, el valor 4= Mucho, se propone:
a)Ampliación del periodo de prácticas ( $88 \%)$
b)Formación de los profesionales colaboradores (79\%).
c)Disponer de guías orientativas detalladas (67\%)
d)Reconocimiento adecuado de la función desarrollada: económica y acadé- micamente $(93 \%)$

e)Mayor colaboración y coordinación con el profesorado tutor de la Universidad $(86 \%)$

Asimismo, valoran que el diseño y desarrollo del Prácticum (estimando únicamente el valor $4=$ Mucho), favorece que el alumnado:

1.Asuma un papel más activo en la construcción del conocimiento y desarrolle habilidades profesionales ( $88 \%)$.

\section{Potencie su autonomía y actitudes colaborativas (92\%).}

3.Incentive su capacidad de valorar críticamente enfoques, resultados y alternativas $(74 \%)$.

4.Facilite la interacción social y profesional con su entorno (91\%).

En relación con la elaboración de la Memoria final, como herramienta más significativa, los profesionales colaboradores que han intervenido en las entrevistas destacan la necesidad de la elaboración del Diario $(89 \%)$, sugiriendo la utilización de las TIC (75\%), frente a la elaboración tradicional; disponer de un diseño/plantilla previos $(91 \%)$; y flexibilizar -según el trabajo realizado y la especialidad- su cumplimentación $(93 \%)$. 


\section{Conclusiones}

Como conclusión global, relativa al objetivo general planteado en la investigación, como aspectos destacados por todos los participantes, se subraya la falta de consideración y reconocimiento académico de las prácticas profesionales, así como la insuficiente relación entre la teoría y la práctica, en la formación de los profesionales estudiados. Igualmente, las propuestas de mejora elaboradas han recalcado la necesidad de potenciar el pensamiento reflexivo y crítico positivo, así como la mejora de la iniciativa personal, la comunicación y el compromiso social con un trabajo bien realizado.

Respecto al objetivo específico a), ha quedado evidente que las metodologías docentes deben favorecer la generación del conocimiento, frente a la simple transmisión de este. Por tanto, se apuesta por el uso de nuevas metodologías (Portafolios y TIC) en las que el alumnado, guiado y motivado por el profesorado, desarrolla un papel fundamental en la adquisición del conocimiento, aprendiendo a aprender. Igualmente, se valora que el profesorado tutor debe poseer grandes dotes humanas y ser capaces de transmitir esa energía activa que estimule en el alumnado un cambio paradigmático, para unir esfuerzos e implementar estrategias efectivas frente al individualismo y la competitividad que nos envuelve en la sociedad actual, a fin de que le posibiliten una efectiva inserción social, desarrollando todas sus capacidades para la convivencia y la autorrealización personal, profesional y laboral. Lógicamente, es fundamental que el profesorado tutor conozca la importancia de la inteligencia emocional en el comportamiento del alumnado y su influencia en las relaciones interpersonales, para propiciar un clima que active la creatividad y generar un espacio de participación en la toma de decisiones (Palomares, 2014).

Como ya se ha indicado, sobre de la duración del periodo de prácticas, el profesorado tutor no está de acuerdo con el periodo estipulado, subrayando la necesidad de ampliarlo. Asimismo, destacan que la división de los periodos (dos en Grado de Maestro y cinco en el Grado de Pedagogía y Grado de Educación Social), sin coordinación entre los mismos genera grandes lagunas y problemas que deberían superarse.

En relación con el objetivo específico b), se coincide con la conclusión de las investigaciones realizadas por Barceló y Ruiz-Corbella (2015), en el sentido que las competencias mejor valoradas por los participantes en los Grados de Maestro son: la capacidad comunicativa y la orientación académica y personal. Sin embargo, en los Grados de Pedagogía y de Educación Social se prioriza la orientación personal y profesional. En la misma línea que los estudios realizados por Cantón, Cañón y Arias (2013), todos los participantes de las titulaciones estudiadas (Grado de Maestro, Pedagogía y Educación Social) evidencian la necesidad de mejorar la formación práctica y acentuar la importancia de una formación integral que articule armónicamente la formación científica y la práctica, desde el inicio de los estudios en la Facultad.

En la investigación se pretendía, como tercer objetivo específico, facilitar la reflexión del alumnado, comprobando que la autoeficacia percibida, entendida como un proceso cognitivo que se produce en el sujeto en interacción con sus experiencias en el desarrollo del Prácticum, es un elemento que precisa ser considerado en próximos trabajos. Por ello, se profundizará en temas como personalidad, la propia motivación de logro, habilidades adquiridas necesarias en comparación con las exigencias de los distintos oficios y tareas, el conocimiento de la oferta formativa y laboral, etc., así como de los contextos y situaciones en los que se desarrollan las prácticas. 
Igualmente, se ha evidenciado la resiliencia como un concepto básico para el desarrollo de la autoeficacia y que se retroalimenta con esta, y se vincula de forma circular con la experiencia, constituyendo un determinante en la formación de la personalidad del sujeto. El desarrollo de una alta resiliencia estará relacionado con una puntuación alta en autoeficacia percibida, puesto que consideramos la resiliencia como la capacidad o el tesón de las personas por persistir en sus conductas a pesar de que los resultados no sean, en principio, del todo favorables para quien las ejecuta. Por lo que, en relación con la autoeficacia percibida, encontraríamos que ante una alta resiliencia existiría -a priori- más probabilidades de obtener distintos resultados fruto del esfuerzo del sujeto en función de la persistencia en conductas resolutivas. Al mismo tiempo, proponemos estudiar la relación entre la percepción de autoeficacia con respecto a las experiencias, ya que ante una espiral de resultados negativos la percepción de control sobre los resultados puede verse afectada generando una sensación de indefensión que, modulada al mismo tiempo por una baja resiliencia, puede ser clave en el desarrollo vocacional y en la elección de una carrera profesional, todo ello fundamental en la figura del profesorado tutor cuando su alumnado está obteniendo el primer contacto con la realidad profesional y laboral de la profesión que han elegido.

Asimismo, dado que una de las limitaciones más significativas del trabajo ha sido la muestra utilizada, resulta inevitable ampliarla al profesorado tutor y a los representantes de las administraciones, de la Universidad de Castilla-la Mancha (UCLM) y la UNED, y comparar los resultados para disponer de evidencias que puedan orientar la elaboración de guías compartidas por todos los implicados en el proceso formativo. Por ello, para los próximos años, se pretende ampliar la investigación a otras Facultades de la UCLM y de la UNED, para profundizar en la investigación, confirmar los resultados y enriquecer -en la medida de lo posible- las propuestas de mejora. 


\section{Bibliografia}

ANECA (2005). Libro Blanco de los títulos de grado en Pedagogía, Magisterio y Educación Social. Madrid: ANECA. http://www.aneca.es/Documentos-y-publicaciones/LibrosBlancos

Barceló, Ma L., \& Ruiz-Corbella, M. (2015). Análisis de las competencias profesionales del Maestro de Educación Primaria. Estudio de Caso. En Ma A. Murga y Ma Paz Trillo (Coord.), Perspectivas y avances de la investigación (pp.35-52). Madrid: UNED.

Campbell, D.T., \& Stanley, J.C. (1978). Diseños experimentales y cuasiexperimentales en la investigación social. Buenos Aires: Amorrortu.

Cantón, I., Cañón, R., \& Arias, A. (2013). La formación universitaria de los maestros de Educación Primaria. Revista interuniversitaria de Formación del Profesorado, $76(27,1)$, 45-63.

Cortina, A. (2017). Ciudadanía europea y multiculturalidad. En V. Climent, F. Michavila, y M. Ripollés (Coord.), Los males de la Europa Social. Buscando soluciones (pp. 191206). Madrid: Tecnos.

De Lara, E., \& Quintanal, J. (Coord.) (2006). El Prácticum en las titulaciones de Educación: Reflexiones y experiencias. Madrid: Dykinson.

Domínguez, J., Álvarez, E., \& López, A. (2017). La violencia escolar en la Educación Secundaria Obligatoria desde la perspectiva del alumnado gallego. Journal of Psychology and Education, 12(2), 95-104. doi: https://doi.org/10.23923/rpye2017.12.148

González, H., Álvarez, J.L., \& Fernández, G. (2012). Fundamentando la formación de los educadores profesionales en competencias interculturales. Bordón. Revista de Pedagogía, 64(1), 89-107.

Guba. E.G. (1989). Criterios de credibilidad en la investigación naturalista. En J. Gimeno y A.I. Pérez Gómez (Eds.), La enseñanza: su teoría y su práctica (pp.148-165). Madrid: Akal.

Juliá, J. (2017). Universidad y economía del conocimiento: dualidad Norte-Sur. En V. Climent, F. Michavila, y M. Ripollés (Coord.), Los males de la Europa Social. Buscando soluciones (pp.243-251). Madrid: Tecnos.

Palomares, A. (2007). Nuevos retos educativos. El modelo docente en el espacio europeo. Cuenca: Universidad de Castilla-La Mancha.

Palomares, A. (2013). La interacción entre utopía y desencanto para una educación de calidad. En Varios, Conversaciones con un Maestro. Homenaje al profesor Dr. José Luis García Garrido (pp.519-526). Madrid: UNED/E. Académicas.

Palomares, A. (2014). Innovative methodologies to promote teacher's emotional competences. Journal for Educators, Teachers and Trainers, 5(2), 116-129. 
Palomares, A. \& Serrano, I. (2016). Influencia de las Competencias Emocionales y la Financiera en la Formación Universitaria. Revista Formación Universitaria, 9 (5), 25-36. doi: $10.4067 /$ S0718-50062016000500004

Pérez Gómez, A.I. (2003). Más allá del academicismo. Los desafíos de la Escuela en la era de la información y la perplejidad. Málaga: Servicio de Publicaciones de la Universidad.

UNESCO (1998). La educación Superior en el Siglo XXI: Visión y Acción. Conferencia Mundial sobre educación Superior. París, 5-9 de octubre.

UNESCO (2017). Educación 2030. Declaración de Incheon y Marco de Acción para la realización del Objetivo de Desarrollo Sostenible 4. Paris: UNESCO. Recuperado de https://goo.gl/gFt5zm 